\title{
Tuning the Hydrolytic Stability of Next Generation Maleimide Cross- Linkers Enables Access to Albumin-Antibody Fragment Conjugates and tri-scFvs
}

\author{
Nafsika Forte, ${ }^{\dagger}$ Maria Livanos, ${ }^{\ddagger}$ Enrique Miranda, ${ }^{\ddagger}$ Maurício Morais, ${ }^{\dagger}$ Xiaoping Yang, ${ }^{\dagger}$

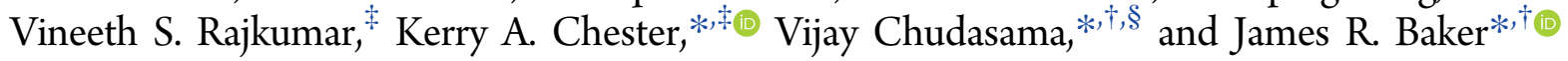 \\ ${ }^{\dagger}$ Department of Chemistry, University College London, 20 Gordon Street, London, WC1H OAJ, United Kingdom \\ ${ }^{*}$ Cancer Institute, University College London, 72 Huntley Street, London, WC1E 6BT, United Kingdom \\ ${ }^{\S}$ Research Institute for Medicines (iMed.ULisboa), Faculty of Pharmacy, Universidade de Lisboa, 1649-004 Lisbon, Portugal
}

Supporting Information

ABSTRACT: We describe investigations to expand the scope of next generation maleimide cross-linkers for the construction of homogeneous protein-protein conjugates. Diiodomaleimides are shown to offer the ideal properties of rapid bioconjugation with reduced hydrolysis, allowing the crosslinking of even sterically hindered systems. The optimized linkers are exploited to link human serum albumin to antibody fragments (Fab or $\mathrm{scFv}$ ) as a prospective half-life extension platform, with retention of antigen binding and robust serum stability. Finally, a triprotein conjugate is formed, by linking $\mathrm{scFv}$ antibody fragments targeting carcinoembryonic antigen. This tri-scFv is shown to infer a combination of greater antigen

avidity and increased in vivo half-life, representing a promising platform for antibody therapeutic development.

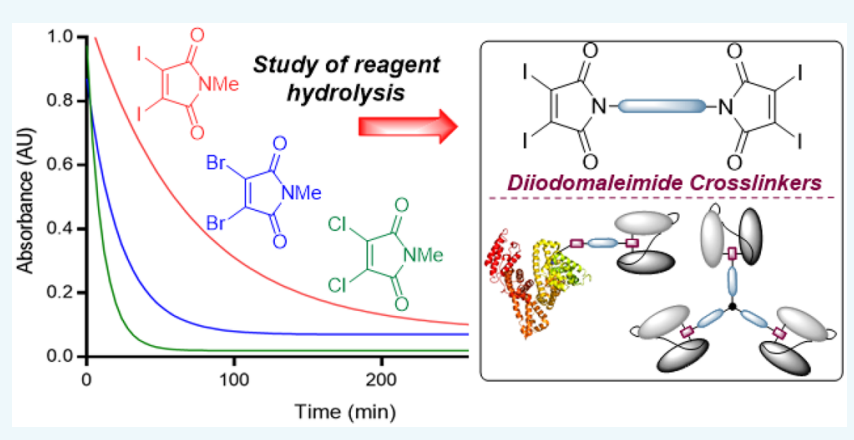

abundance, lysine modification yields heterogeneous products with inconsistent pharmacological profiles and toxicities. ${ }^{10,11}$ In contrast, cysteine residues are rare in proteins and are often found tied up in disulfide bonds. ${ }^{12}$ Selective modification can be achieved upon disulfide bond reduction and subsequent conjugation; however, the structural integrity of the protein is often compromised. ${ }^{13,14}$ In addition, due to the presence of two cysteine residues per disulfide bond, it is not possible to obtain a homogeneous $1: 1$ protein-protein conjugate using classical maleimide chemistry. ${ }^{15}$ Recent advances in bioconjugation have shown that cysteine-based site-directed mutagenesis or unnatural amino acid insertion can offer a solution to the issue of chemoselectivity. ${ }^{16}$ For example, using the latter approach Schultz et al. have reported on the formation of various anti-HER2/anti-CD3 bispecific antibodies in good yields. ${ }^{17}$ While this method is elegant, it is not always readily transferable; laborious screening procedures are required to identify suitable positions for modification and substitution for the unnatural amino acids can often be incomplete. ${ }^{18,19}$ Thus, site-selective methods that are directly applicable to native proteins and avoid the need for genetic engineering remain highly desirable.

Received: December 13, 2017

Revised: January 11, 2018

Published: January 31, 2018 
The functional rebridging of native disulfide bonds represents a highly promising strategy to achieving homogeneous and structurally intact protein conjugates. We have previously reported on the use of next generation maleimides $(\mathrm{NGMs})^{20-22}$ and pyridazinediones (PDs) ${ }^{23-27}$ as highly efficient disulfide bridging reagents. Most notably, we have shown that a bis-dibromomaleimide PEG cross-linker $\left(\mathbf{D B M}_{\mathrm{CL}}\right.$ Figure 1) can be used to create a bispecific scFv-Fab antibody

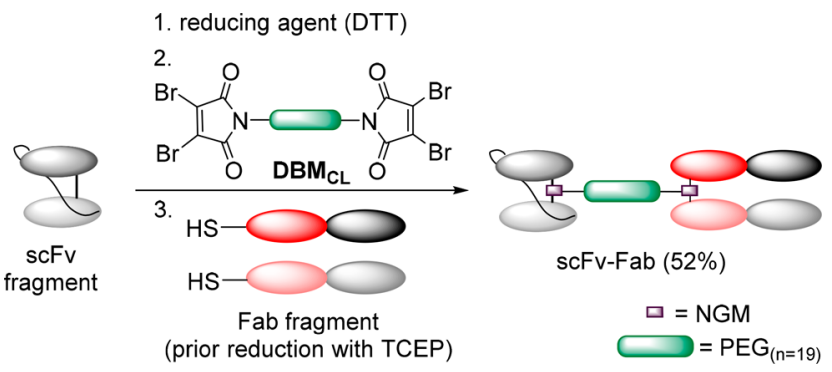

Figure 1. Generation of scFv-Fab bispecific by disulfide bridging using a bis-dibromomaleimide PEG cross-linking reagent $\left(\mathbf{D B M}_{\mathrm{CL}}\right)$. $^{28}$

in $52 \%$ isolated yield, in a site-specific and homogeneous manner. ${ }^{28}$ In addition, relevant work in this area carried out by Brocchini et al. has shown that by using a PEG-di(bis-sulfone) reagent, two Fab antibody fragments can be linked together to create an IgG mimetic by disulfide bridging, albeit in lower yield $(12 \%){ }^{29}$

In this work, we were interested in extending the application of these NGM cross-linkers. We identified several challenges which would further test the scope of the methodology, including conjugating proteins containing different reactive handles (i.e., a single cysteine to a disulfide), reacting with sterically hindered conjugation sites, generating proteinprotein conjugates which are robustly stable in serum and finally linking together three proteins using trifunctional crosslinkers.

To address these challenges we selected Human Serum Albumin (HSA) as the ideal cysteine containing protein, since it contains a single free thiol (Cys-34) available for conjugation. ${ }^{30,31}$ The literature suggests that even though the direct chemical conjugation of HSA to peptides is feasible, there is a clear challenge in conjugating bigger biomolecules. This can be attributed to Cys-34 being positioned within a $9.5 \AA$ crevice, which diminishes its solvent accessibility and hence reactivity. ${ }^{32}$ HSA has proven to be a valuable clinical tool for extending the circulatory life span of attached proteins to provide prolonged therapeutic cover. ${ }^{33}$ This is due to a combination of its large size (ca. $66.5 \mathrm{kDa}$ ) that is above the renal threshold and recycling via the neonatal $\mathrm{Fc}$ receptor $(\mathrm{FcRn})$. For example, there has been considerable interest in the generation of albumin-antibody fragment constructs for therapeutic or imaging applications with the products exhibiting significantly increased half-lives in vivo and enhanced tumor-to-blood ratios. ${ }^{34}$ Previous approaches to such constructs have relied on either fusion, ${ }^{35}$ which is not ideal since it can result in reduction of HSA binding to the FcRn receptor, ${ }^{36}$ or suboptimal chemical conjugation affording low yields of the target conjugate. ${ }^{37}$

We chose to evaluate the application of NGM bifunctional reagents toward the synthesis of albumin-antibody fragment conjugates by employing two clinically relevant fragments that contain a single disulfide bond; trastuzumab (Herceptin) Fab, which targets the HER2/neu receptor overexpressed in 25$30 \%$ of breast cancers, ${ }^{38}$ and anti-CEA scFv that targets the Carcinoembryonic antigen (CEA) present in a wide range of cancers (particularly colorectal carcinoma). ${ }^{39}$ We also aimed to develop trifunctional NGM reagents and apply them to the construction of protein triconjugates. We identified tri-scFvs as being ideal targets since they are considered within an optimal "tumor-target zone", prospectively offering a good balance between reduced clearance rates (compared to scFvs) and increased tumor penetration (compared to intact immunoglobulins). ${ }^{40,41}$

In prior work we had demonstrated that the $\mathrm{DBM}_{\mathrm{CL}}$ reagent was very efficient in linking $\mathrm{scFv}$ and $\mathrm{Fab}$ fragments. ${ }^{28} \mathrm{We}$ began our study toward the synthesis of albumin-antibody fragment conjugates, by initially employing this reagent. The choice of a homobifunctional reagent for the conjugation of different reactive centers (cysteine to disulfide) was made to preclude issues of regioselectivity. HSA was shown to successfully react with the $\mathrm{DBM}_{\mathrm{CL}}$; however upon addition of reduced $\mathrm{scFv}$ or $\mathrm{Fab}$ to attempt the cross-linking, only a small amount of product could be observed by SDS-PAGE, which did not increase by varying conditions (see SI Figures S7-S8). We hypothesized that the increased steric hindrance of HSA must be inhibiting the bioconjugation. Over time, hydrolysis of the second DBM motif to an unreactive dibromomaleamic acid can become a competing process, preventing the conjugation. ${ }^{42}$ The susceptibility of maleimides toward hydrolysis, and the effect that $N$-substitution has on its rate, has been well reported in the literature. ${ }^{9,43,44}$ In order to gain a better understanding of the NGM platform and hence develop robust cross-linking reagents, it was deemed necessary to study the influence that the leaving groups have on the rate of hydrolysis. This was achieved by incubating $N$-methyl maleimides possessing different halogen leaving groups at $\mathrm{pH} 7.4$ and monitoring their absorbance at ca. $300-400 \mathrm{~nm}$ over time, which is lost upon conversion to maleamic acids (Figure 2). ${ }^{42}$ A direct correlation between increasing electronegativity of the leaving

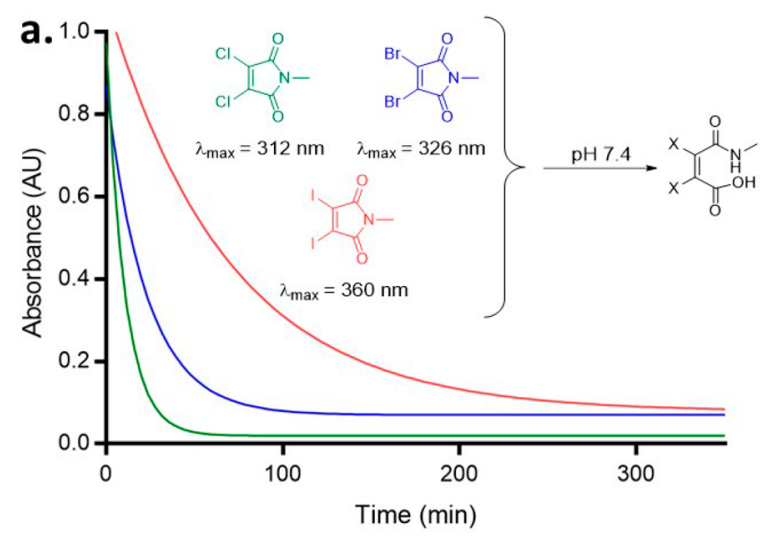

\begin{tabular}{c|c|c} 
b. Substituent, $\mathbf{X}$ & $\mathrm{K}_{1, \text { obs }}\left(\mathbf{m i n}^{-1}\right)$ & Hydrolysis $\mathrm{t}_{1 / 2}(\mathrm{~min})$ \\
\hline $\mathrm{Cl}$ & 0.07659 & 9.1 \\
\hline $\mathrm{Br}$ & 0.03871 & 17.9 \\
\hline $\mathrm{I}$ & 0.01444 & 48.0
\end{tabular}

Figure 2. Hydrolysis of $\mathrm{N}$-methyl halomaleimide derivatives over time: a, UV/vis absorbance over time; b, determination of pseudo-first-order rate constants $\left(k_{1, \mathrm{obs}}\right)$ and hydrolysis half-life $\left(t_{1 / 2}\right)$. 
Scheme 1. Synthesis of Bifunctional Diiodomaleimide Cross-Linking Reagents ${ }^{a}$

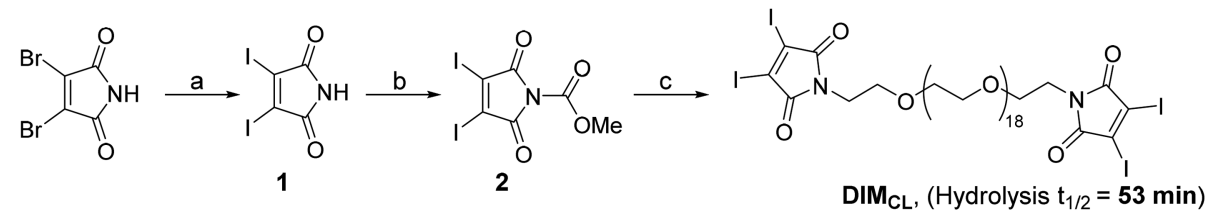

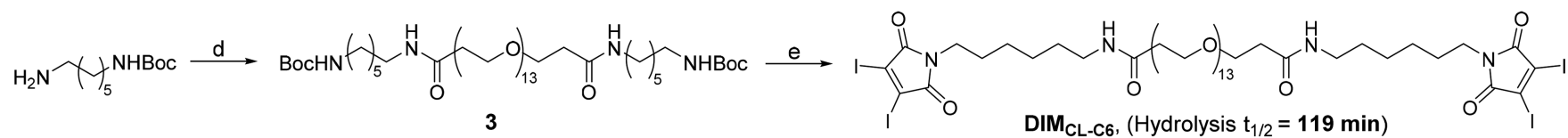

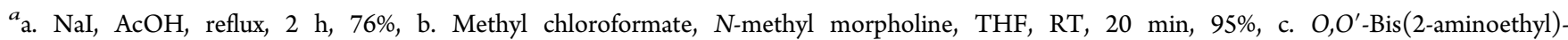
octadecaethylene glycol, DCM, silica, RT, 20 h, 66\%, d. O,O'-Bis (2-carboxyethyl)dodecaethylene glycol, EDC.HCl, DMAP, HOBt, DMF, $0{ }^{\circ} \mathrm{C}$ to RT, 20 h, 79\%, e. (i) TFA/DCM, RT, 12 h then DIPEA, DCM, RT, 15 min, (ii) N-(methoxycarbonyl)-3,4-diiodomaleimide 2, RT, 1 h, then $p$ TsOH, RT, 18 h, $67 \%$.

a.

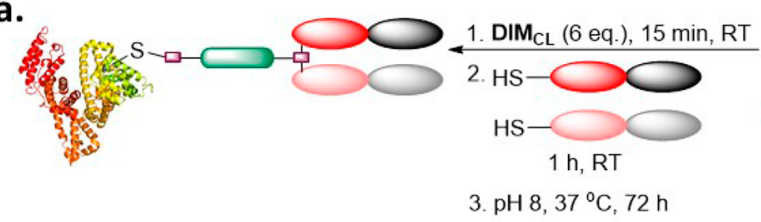

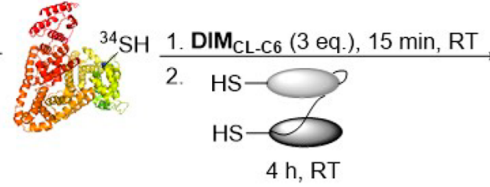

3. $\mathrm{pH} 8,37^{\circ} \mathrm{C}, 72 \mathrm{~h}$

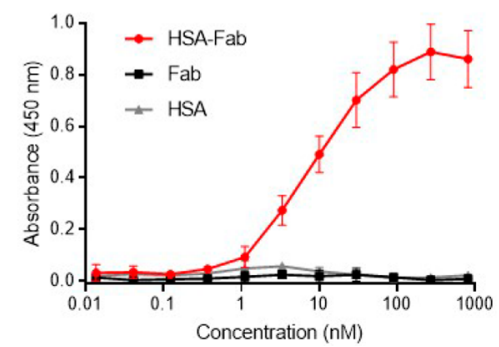

d.

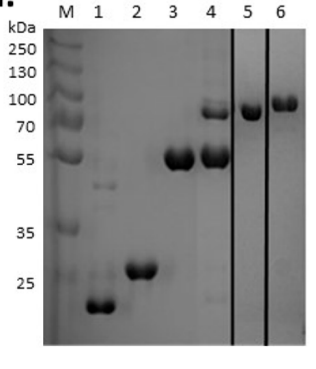

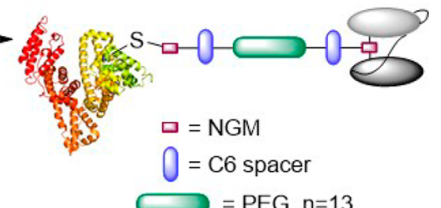

e.

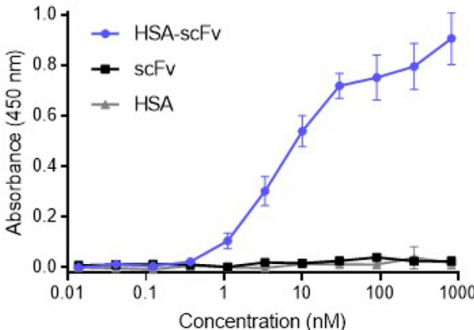

Figure 3. a. HSA-Fab and HSA-scFv conjugation reaction scheme. b. SDS-PAGE analysis of HSA-Fab conjugation: M, Molecular marker; 1, Native Fab; 2, Reduced Fab (10 equiv TCEP, 1 h, $37^{\circ} \mathrm{C}$ ); 3, Native HSA; 4, HSA-Fab conjugation mixture (3:1) in buffer containing $500 \mathrm{mM} \mathrm{NaCl;} 5$, Purified HSA-Fab conjugate; 6, Purified HSA-Fab conjugate (reducing gel). c. Sandwich ELISA analysis of HSA-Fab, native HSA, and native Fab. d. SDS-PAGE analysis of HSA-scFv conjugation: M, Molecular marker; 1, Native scFv; 2, Reduced scFv (20 equiv DTT, 1 h, RT); 3, Native HSA; 4, HSA-scFv conjugation mixture (3:1); 5, Purified HSA-scFv conjugate; 6, Purified HSA-scFv conjugate (reducing gel); e, Sandwich ELISA analysis of HSA-scFv, native HSA, and native scFv.

group and hydrolysis rate could be clearly observed, consistent with the inductive effect activating the carbonyls to nucleophilic attack. Notably dibromomaleimide exhibited a short half-life of $17.9 \mathrm{~min}$, which likely explains the poor outcome in the case of the sterically encumbered HSA-antibody fragment conjugation.

We focused our attention on the use of iodine substituents with the view to developing robust NGM cross-linking reagents. The diiodomaleimide (DIM) was shown to hydrolyze more slowly than the DBM by a factor of 2.7 , as evidenced by the UV study (see Figure 2). In addition, we have previously observed, through stopped-flow analysis, DIM to be a faster conjugation reagent than DBM, suggesting that the decreased mesomeric donation from iodine into the maleimide ring makes it a better conjugate acceptor. ${ }^{45}$

We synthesized two bis-DIM PEG cross-linking reagents: one that also incorporated a C6 spacer between maleimide and PEG to further increase the hydrolytic stability (reagents DIM $_{\text {CL }}$ and DIM CL-C6 $_{\text {6 }}$ Scheme 1). The synthesis involved an initial Finkelstein-type reaction to afford diiodomaleimide 1, from commercially available dibromomaleimide. This was then activated with an $\mathrm{N}$-methoxycarbonyl to give 2 , and reacted with either commercially available PEG(19) diamine or synthesized C6-PEG(13) diamine 3, respectively. With both cross-linkers in hand we set out to explore the conjugation of HSA to Fab and scFv. We were delighted to observe that conjugation of the Fab to HSA was highly efficient with either linker used. To this end, we chose the reagent with the PEG spacer to carry out further optimization. In contrast the $\mathrm{scFv}$, which contains a more hindered disulfide, necessitated the use of the more hydrolytically stable $\mathbf{D I M}_{\mathrm{CL}-\mathrm{C} 6}$ linker in order to achieve high conversion to the target conjugate.

The optimized conjugation protocols involved HSA initially reacting with either linker in excess for $15 \mathrm{~min}$ at room temperature, with no dimerization of HSA observed under these conditions. Following removal of the excess reagent via ultrafiltration, reduced $\mathrm{Fab}$ or $\mathrm{scFv}$ were added into the conjugation in a 3:1 ratio ( 3 equiv HSA to 1 equiv $\mathrm{Fab}$ or $\mathrm{scFv}$ ) at $74.4 \mu \mathrm{M}$. Complete consumption of $\mathrm{Fab}$ and $\mathrm{scFv}$ was observed by SDS-PAGE analysis after 1 and $4 \mathrm{~h}$ of incubation at $\mathrm{pH} 7.4$, respectively (Figure 3b, lane 4; Figure 3d, lane 4). Interestingly, during optimization of the HSA-Fab conjugation, the presence of a second species at $\mathrm{ca} .70 \mathrm{kDa}$ was observed on 


\section{Scheme 2. Synthesis of Trifunctional Diiodomaleimide Cross-Linking Reagent ${ }^{a}$}

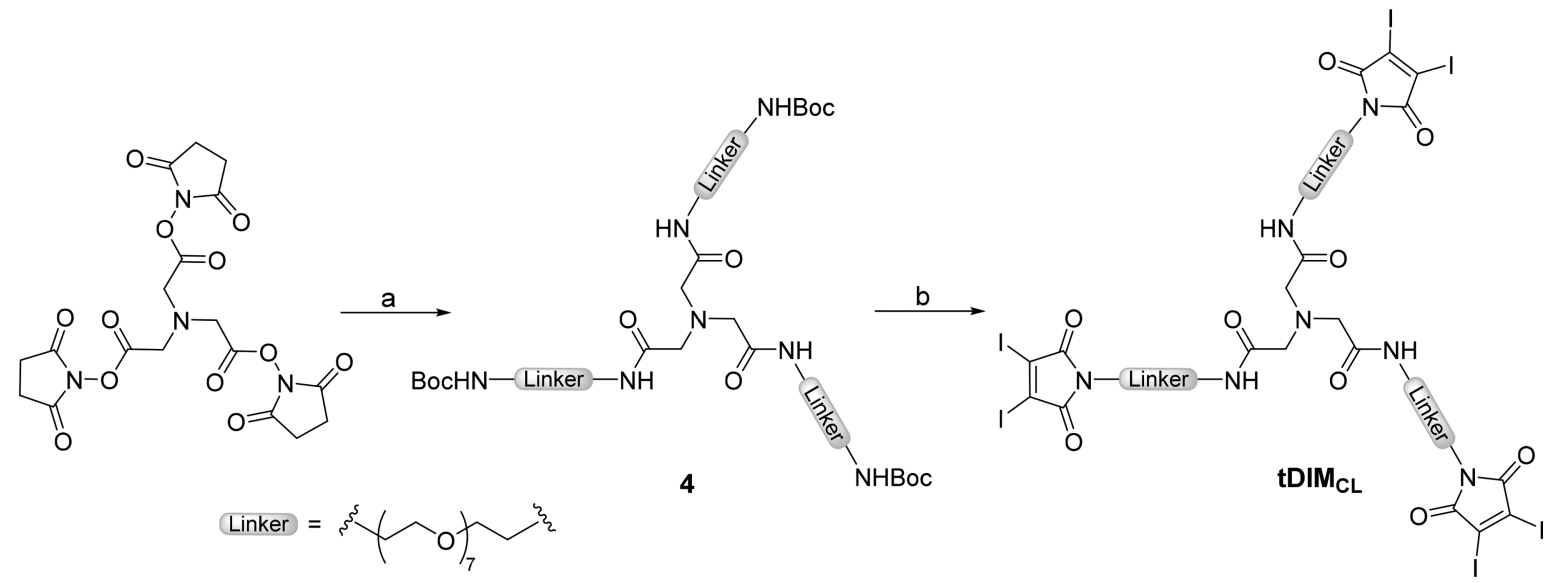

$a_{\text {a. }}$ O-(2-Aminoethyl)-O'-[2-(Boc-amino)ethyl] hexaethylene glycol, RT, 20 h, 81\%; b. (i) TFA/DCM, then $\mathrm{H}_{2} \mathrm{O}$, pH 11, extracted into DCM; (ii) $N$-(methoxycarbonyl)-3,4-diiodomaleimide 2, DCM, RT, 2 h, then silica, RT, 1 h, 68\%.

a.
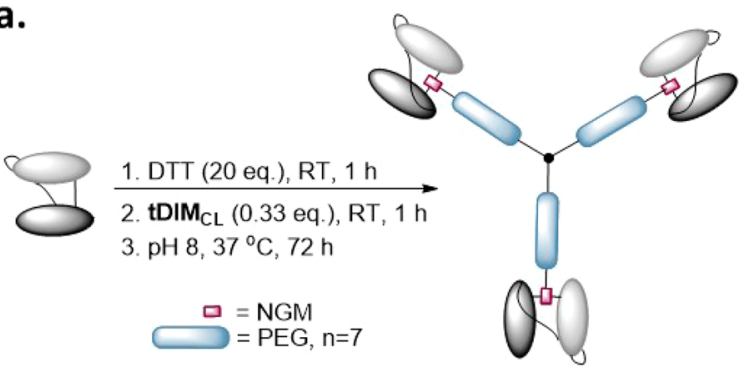

b.

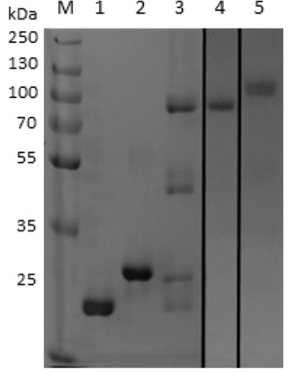

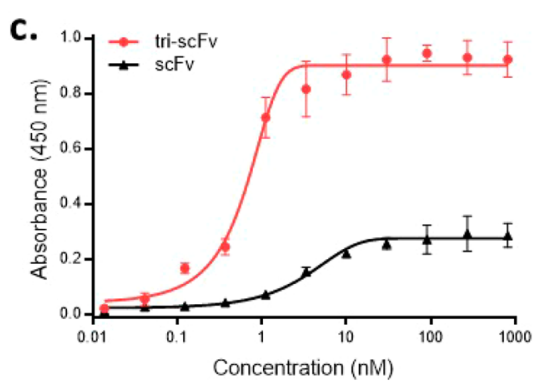

d. $B A L B \backslash C$ monomer Biodistribution

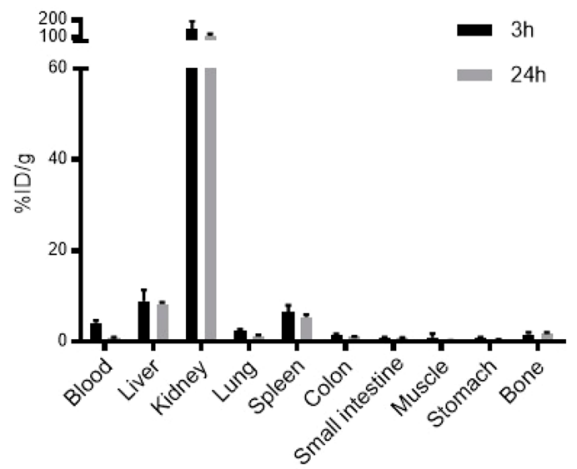

e. $B A L B \backslash C$ trimer Biodistribution

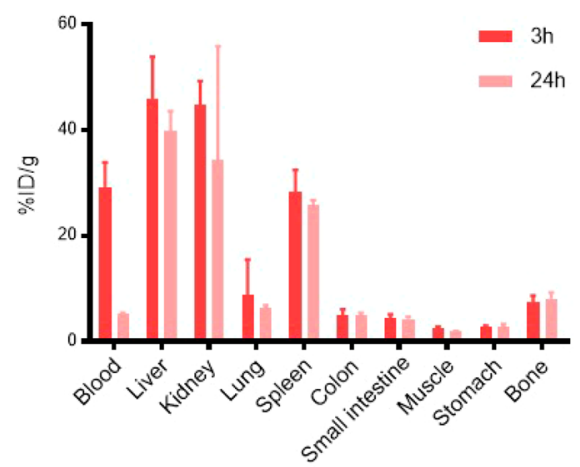

f.

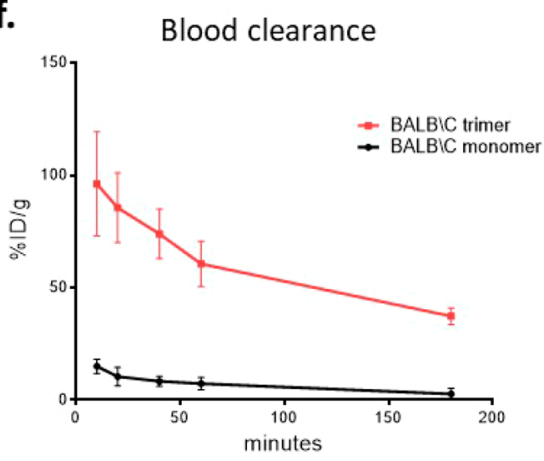

Figure 4. a. Tri-scFv conjugation reaction scheme. b. SDS-PAGE analysis of tri-scFv conjugation: M, Molecular marker; $1, \mathrm{Native} s \mathrm{scF}$; 2 , Reduced scFv (20 equiv DTT, 1 h, RT); 3, tri-scFv conjugation mixture; 4, Purified tri-scFv conjugate; 5, Purified tri-scFv conjugate (reducing gel). c. ELISA analysis of tri-scFv and scFv against full length CEA. d. Biodistribution of ${ }^{111}$ In-labeled scFv in BALB $\backslash \mathrm{C}$ mice at 3 and 24 h. e. Biodistribution of ${ }^{111}$ In-labeled tri-scFv in BALB $\backslash \mathrm{C}$ mice at 3 and $24 \mathrm{~h}$. f. Blood clearance of tri-scFv and scFv in BALB $\backslash \mathrm{C}$ mice. Each data point represents the mean of 3 mice \pm SD.

the gel, which corresponded to the conjugate lacking a covalent linkage to the Fab light chain (see SI Figures 12-13). This incomplete disulfide bridging was solved by performing the conjugation in buffer containing increased salt concentration (i.e., $500 \mathrm{mM} \mathrm{NaCl}$ ) with the view to disrupting any noncovalent interactions that were inhibiting reaction of the light chain cysteine (Figure 3b, lane 4).

Following conjugation, both reaction mixtures were incubated at $\mathrm{pH} 8.0$ for $72 \mathrm{~h}$ at $37{ }^{\circ} \mathrm{C}$ to effect hydrolysis in order to obtain serum stable conjugates. ${ }^{46}$ The mixtures were then purified to afford HSA-Fab and HSA-scFv in $49 \%$ and $57 \%$ yield, with respect to the antibody fragment limiting reagents. These yields represent a significant improvement over previous reported yields on the direct conjugation of albumin to larger proteins. ${ }^{37,47}$

Both HSA-Fab and HSA-scFv conjugates were shown to retain full binding activity to HER2 and CEA antigens, respectively, as determined by ELISA (see SI). In addition, a sandwich ELISA was developed, in order to demonstrate the bivalent nature of the two conjugates; this involved binding to HER2 or CEA, followed by detection with an antialbumin antibody (see SI for further details). As expected, only the 
HSA-Fab and HSA-scFv conjugates gave a signal, since they can bind simultaneously to the antigen and antialbumin (Figure $3 c$ and e). In addition, no evidence of cleavage or aggregation was observed upon incubation in human serum at $37^{\circ} \mathrm{C}$ for 1 week or storage at $-20{ }^{\circ} \mathrm{C}$ for 9 months (see SI for further details).

The successful synthesis of conjugates was also confirmed by LC-MS (see SI Figures S11 and S18). Interestingly, LC-MS analysis revealed the loss of the second iodine from the maleimide motif that had reacted with HSA. To gain a better insight into the reaction and simplify analysis, we reacted HSA with $\mathrm{N}$-Me diiodo-, dibromo-, and dichloromaleimide. All dihalomaleimides were shown to react with HSA in identical manner, i.e., the second halogen was not present, which led us to postulate that cross-linking with a nucleophilic residue nearby Cys-34 had taken place. To identify this point of attachment, we performed a tryptic digest of HSA modified with commercially available $\mathrm{N}-\mathrm{Me}$ dibromomaleimide, as well as a synthesized $\mathrm{N}$-biotin dibromomaleimide. These analyzed conjugates were constructed under the same conditions as the cross-linked conjugates, including incubation at $\mathrm{pH} 8,37^{\circ} \mathrm{C}$, for $72 \mathrm{~h}$ to infer thiol stability which was essential due to the presence of DTT in the digest protocol (see SI). LC-MS and LC-MS/MS analysis of the peptide mixtures post digestion revealed the point of cross-linking to be Lysine-41 (see SI). This is consistent with its position $9.5 \AA$ from Cys-34 (see SI Figure S38, PDB file 1AO6).

Having successfully identified the appropriate NGM linker design to promote conjugation of two proteins in a sterically hindered system, we wished to extend our technology to allow for the creation of a triprotein conjugate. Rapidly clearing antibody fragments are considered ideal for tumor imaging, because they can penetrate tumors well and generate high tumor to background ratios. However, for tumor therapy, such fragments are unlikely to give sustained effects due to the high target retention required. ${ }^{48}$ Efforts to reduce blood clearance have been made by conjugating $\mathrm{scFv}$ monomers into multivalent constructs of increased avidity and size. ${ }^{49} \mathrm{scFv}$ dimers have exhibited increased tumor uptake compared to monomers, but since their size (ca. $55 \mathrm{kDa}$ ) is just below the renal threshold, their circulating half-life has only been shown to increase by a factor of ca. 2.5.

Higher multimers, such as scFv triabodies (ca. $90 \mathrm{kDa}$ ) have attracted considerable interest recently; their optimal size precludes fast renal clearance. ${ }^{41}$ In addition, the trivalency can confer a significant increase in avidity, which is particularly beneficial for low affinity binders, since poor retention can result in diffusion out of the tumor tissue, requiring higher concentrations of the antibody in order to bind a significant fraction of the antigen. ${ }^{41}$

We aimed at developing an efficient one-pot procedure to generate a homotrimer of the low binder anti-CEA shmFe scFv, by disulfide bridging using the tris-diiodomaleimide PEG crosslinker tDIM $_{\mathrm{CL}}$, which was synthesized over two steps (Scheme 2 ). The synthesis commenced with a coupling between the commercially available tri-NHS ester and PEG(7) mono-Boc protected diamine to afford the trifunctional scaffold 4 . After Boc deprotection, this was reacted with $N$-methoxycarbonyl diiodomaleimide 2 to afford trilinker $\mathbf{t D I M}_{\mathbf{C L}}$.

The optimized conjugation protocol involved initial reduction of the $\mathrm{scFv}$ (20 equiv DTT, RT, $1 \mathrm{~h}$ ), which after removal of excess DTT was incubated with 0.33 equiv of linker $\mathbf{t D I M}_{\mathrm{CL}}$ at $37.2 \mu \mathrm{M}$ (Figure 4). Pleasingly, after $1 \mathrm{~h}$ a strong band corresponding to the target trimer could be observed by SDS-
PAGE analysis (Figure $4 \mathrm{~b}$, lane 3 ). The reaction mixture was then buffer exchanged to $\mathrm{pH} 8.0$ buffer and incubated for $72 \mathrm{~h}$ at $37{ }^{\circ} \mathrm{C}$ to promote hydrolysis in order to obtain a serum stable construct. Subsequent purification by size exclusion chromatography afforded tri-scFv in $30 \%$ yield, the successful formation of which was confirmed by LC-MS (Figure S22). ELISA analysis of the conjugate (Figure $4 c$ ) demonstrated that trimerization has successfully led to a decrease in the $\mathrm{IC}_{50}\left(\mathrm{IC}_{50}\right.$ $0.52 \mathrm{nM}$ for trimer vs $\mathrm{IC}_{50} 4.18 \mathrm{nM}$ for monomer).

Having successfully synthesized anti-CEA tri-scFv, we wanted to evaluate its biodistribution in BALB $\backslash \mathrm{C}$ mice, compared to the unmodified monomer, in order to establish whether the increased molecular weight had conferred slower blood clearance. Tri-scFv and $\mathrm{scFv}$ were conjugated to $\mathrm{N}$ hydroxysuccinimidyl DOTA for radiolabeling with $\left[{ }^{111} \mathrm{In}\right]$. ELISA analysis after DOTA conjugation demonstrated that immunoreactivity was identical to that before conjugation (see Figure S27). In addition, size exclusion chromatograms of triscFv and $\mathrm{scFv}$ obtained before and after DOTA-labeling confirmed that the integrity of the conjugates had been maintained. Radiolabeling with $\left[{ }^{111} \mathrm{In}\right]$ was achieved with $97 \%$ incorporation efficiency. Groups of three mice were sacrificed at 3 and $24 \mathrm{~h}$; the tissues were analyzed and the data for both conjugates plotted as \% ID/g (Figure $4 \mathrm{~d}-\mathrm{f}$ ). The monomer demonstrated rapid uptake in the kidney, reaching $149.2 \% \pm$ $45.4 \% \mathrm{ID} / \mathrm{g}$ in $3 \mathrm{~h}$ (Figure $4 \mathrm{~d}$ ). The blood clearance was shown to be extremely fast (Figure $4 \mathrm{f}$ ), with the blood level of activity at $4.2 \% \pm 0.5 \% \mathrm{ID} / \mathrm{g}$ in $3 \mathrm{~h}$. In contrast, tri-scFv showed a much more even distribution (Figure 4e). Its blood clearance was markedly reduced (Figure 4f) compared to the monomer, with the blood level exhibiting a 7 -fold increase $(29.0 \% \pm 4.8 \%$ ID/g in $3 \mathrm{~h}$ ). Kidney accumulation was also observed after $3 \mathrm{~h}$, with the level of activity being at $44.7 \% \pm 4.6 \% \mathrm{ID} / \mathrm{g}$ (ca. $3 \times$ lower than the monomer); however, this seems to be consistent with previous results observed for fragments labeled with metallic isotopes, such as ${ }^{111} \mathrm{In} .{ }^{50}$

\section{CONCLUSION}

Diiodomaleimide cross-linking reagents have been demonstrated to offer an optimized platform for the construction of homogeneous and stable protein-protein conjugates. Due to their increased hydrolytic stability and rapid reactivity with cysteine residues they enabled the conjugation of sterically hindered protein systems, such as HSA to an scFv antibody fragment, in a highly efficient manner. The products were obtained in good yields, were stable in serum and retained full binding affinity to the target antigens. These protein conjugates also demonstrate the viability of cross-linking between different reactive handles using homobifunctional NGMs, a cysteine on one protein to a disulfide bond on another.

The synthesis of a trifunctional diiodomaleimide conjugation reagent was also developed, which enabled the generation of an anti-CEA scFv homotrimer in an efficient one-pot procedure. The trimerization of $\mathrm{scFv}$ by disulfide bridging was shown to significantly increase the circulatory half-life of the construct, compared to the unmodified monomer. Overall, we believe that the slower blood clearance of tri-scFv, in combination with the increase in valency could lead to the development of powerful constructs for in vivo applications. 


\section{ASSOCIATED CONTENT}

\section{S Supporting Information}

The Supporting Information is available free of charge on the ACS Publications website at DOI: 10.1021/acs.bioconjchem.7b00795.

Full experimental procedures and data (PDF)

\section{AUTHOR INFORMATION}

\section{Corresponding Authors}

*E-mail: k.chester@ucl.ac.uk.

*E-mail: v.chudasama@ucl.ac.uk.

*E-mail: j.r.baker@ucl.ac.uk.

\section{ORCID}

Kerry A. Chester: 0000-0002-3093-3130

James R. Baker: 0000-0002-7223-2279

Notes

The authors declare no competing financial interest.

\section{ACKNOWLEDGMENTS}

We gratefully acknowledge BBSRC and EPSRC, for funding and the EPSRC UK National Mass Spectrometry Facility (NMSF), Swansea. We also thank Dr. Kersti Karu and Dr. Abil Aliev for assistance with mass spectrometry and NMR analysis, respectively.

\section{REFERENCES}

(1) Kontermann, R. E. (2012) Dual targeting strategies with bispecific antibodies. MAbs 4, 182-197.

(2) Cao, Y., and Lam, L. (2003) Bispecific antibody conjugates in therapeutics. Adv. Drug Delivery Rev. 55, 171-197.

(3) Yu, K., Liu, C., Kim, B.-G., and Lee, D.-Y. (2015) Synthetic fusion protein design and applications. Biotechnol. Adv. 33, 155-164.

(4) Santagostino, E. (2016) Transforming the treatment for hemophilia B patients: update on the clinical development of recombinant fusion protein linking recombinant coagulation factor IX with recombinant albumin (rIX-FP). Thromb. Res. 141, S5-S8.

(5) Baeuerle, P. A., and Reinhardt, C. (2009) Bispecific T-cell engaging antibodies for cancer therapy. Cancer Res. 69, 4941-4944.

(6) Dreier, T., Lorenczewski, G., Brandl, C., Hoffmann, P., Syring, U., Hanakam, F., Kufer, P., Riethmuller, G., Bargou, R., and Baeuerle, P. A. (2002) Extremely potent, rapid and costimulation-independent cytotoxic T-cell response against lymphoma cells catalyzed by a single-chain bispecific antibody. Int. J. Cancer 100, 690-697.

(7) Chames, P., and Baty, D. (2009) Bispecific antibodies for cancer therapy: the light at the end of the tunnel? MAbs 1, 539-547.

(8) Schmidt, S. R. (2009) Fusion-proteins as biopharmaceuticals Applications and challenges. Curent Opin. Drug Discovery 12, 284-295.

(9) Hermanson, G. T. (2008) Bioconjugate Techniques, 2nd ed. Academic Press: London, UK.

(10) Wang, L., Amphlett, G., Blattler, W. A., Lambert, J. M., and Zhang, W. (2005) Structural characterization of the maytansinoidmonoclonal antibody immunoconjugate, huN901-DM1, by mass spectrometry. Protein Sci. 14, 2436-2446.

(11) Junutula, J. R., Raab, H., Clark, S., Bhakta, S., Leipold, D. D., Weir, S., Chen, Y., Simpson, M., Tsai, S. P., Dennis, M. S., et al. (2008) Site-specific conjugation of a cytotoxic drug to an antibody improves the therapeutic index. Nat. Biotechnol. 26, 925-932.

(12) Fodje, M. N., and Al-Karadaghi, S. (2002) Occurrence, conformational features and amino acid propensities for the pi-helix. Protein Eng., Des. Sel. 15, 353-358.

(13) Hamblett, K. J., Senter, P. D., Chace, D. F., Sun, M. M. C., Lenox, J., Cerveny, C. G., Kissler, K. M., Bernhardt, S. X., Kopcha, A. K., Zabinski, R. F., et al. (2004) Effects of Drug Loading on the Antitumor Activity of a Monoclonal Antibody Drug Conjugate. Clin. Cancer Res. 10, 7063-7070.
(14) Adem, Y. T., Schwarz, K. A., Duenas, E., Patapoff, T. W., Galush, W. J., and Esue, O. (2014) Auristatin antibody drug conjugate physical instability and the role of drug payload. Bioconjugate Chem. 25, 656664.

(15) Glennie, M. J., Mcbride, H. M., Worth, A. T., and Stevenson, G. T. (2017) Preparation and performance of bispecific F(ab' gamma)2 antibody containing thioether-linked Fab' gamma fragments. $J$. Immunol. 139, 2367-2375.

(16) Chalker, J. M., Bernardes, G. J. L., Lin, Y. A., and Davis, B. G. (2009) Chemical modification of proteins at cysteine: opportunities in chemistry and biology. Chem. - Asian J. 4, 630-640.

(17) Cao, Y., Axup, J. Y., Ma, J. S. Y., Wang, R. E., Choi, S., Tardif, V., Lim, R. K. V., Pugh, H. M., Lawson, B. R., Welzel, G., et al. (2015) Multiformat T-Cell-Engaging Bispecific Antibodies Targeting Human Breast Cancers. Angew. Chem., Int. Ed. 54, 7022-7027.

(18) Loscha, K. V., Herlt, A. J., Qi, R., Huber, T., Ozawa, K., and Otting, G. (2012) Multiple-site labeling of proteins with unnatural amino acids. Angew. Chem., Int. Ed. 51, 2243-2246.

(19) Johnson, D. B. F., Xu, J., Shen, Z., Takimoto, J. K., Schultz, M. D., Schmitz, R. J., Ecker, J. R., Briggs, S. P., and Wang, L. (2011) RF1 knockout allows ribosomal incorporation of unnatural amino acids at multiple sites. Nat. Chem. Biol. 7, 779-786.

(20) Smith, M. E. B., Schumacher, F. F., Ryan, C. P., Tedaldi, L. M., Papaioannou, D., Waksman, G., Caddick, S., and Baker, J. R. (2010) Protein modification, bioconjugation, and disulfide bridging using bromomaleimides. J. Am. Chem. Soc. 132, 1960-1965.

(21) Schumacher, F. F., Sanchania, V. A., Tolner, B., Wright, Z. V. F., Ryan, C. P., Smith, M. E. B., Ward, J. M., Caddick, S., Kay, C. W. M., Aeppli, G., et al. (2013) Homogeneous antibody fragment conjugation by disulfide bridging introduces "spinostics. Sci. Rep. 3, 1525.

(22) Marculescu, C., Kossen, H., Morgan, R. E., Mayer, P., Fletcher, S. A., Tolner, B., Chester, K. A., Jones, L. H., and Baker, J. R. (2014) Aryloxymaleimides for cysteine modification, disulfide bridging and the dual functionalization of disulfide bonds. Chem. Commun. 50, $7139-7142$.

(23) Chudasama, V., Smith, M. E. B., Schumacher, F. F., Papaioannou, D., Waksman, G., Baker, J. R., and Caddick, S. (2011) Bromopyridazinedione-mediated protein and peptide bioconjugation. Chem. Commun. 47, 8781-8783.

(24) Maruani, A., Smith, M. E. B., Miranda, E., Chester, K. A., Chudasama, V., and Caddick, S. (2015) A plug-and-play approach to antibody-based therapeutics via a chemoselective dual click strategy. Nat. Commun. 6, 6645 .

(25) Robinson, E., Nunes, J. P. M., Vassileva, V., Maruani, A., Nogueira, J. C. F., Smith, M. E. B., Pedley, R. B., Caddick, S., Baker, J. R, and Chudasama, V. (2017) Pyridazinediones deliver potent, stable, targeted and efficacious antibody-drug conjugates (ADCs) with a controlled loading of 4 drugs per antibody. RSC Adv. 7, 9073-9077.

(26) Lee, M. T. W., Maruani, A., Baker, J. R., Caddick, S., and Chudasama, V. (2016) Next-generation disulfide stapling: reduction and functional re-bridging all in one. Chem. Sci. 7, 799-802.

(27) Lee, M. T. W., Maruani, A., Richards, D. A., Baker, J. R, Caddick, S., and Chudasama, V. (2017) Enabling the controlled assembly of antibody conjugates with a loading of two modules without antibody engineering. Chem. Sci. 8, 2056-2060.

(28) Hull, E. A., Livanos, M., Miranda, E., Smith, M. E. B., Chester, K. A., and Baker, J. R. (2014) Homogeneous Bispecifics by Disulfide Bridging. Bioconjugate Chem. 25, 1395-1401.

(29) Khalili, H., Godwin, A., Choi, J.-W., Lever, R., Khaw, P. T., and Brocchini, S. (2013) Fab-PEG-Fab as a potential antibody mimetic. Bioconjugate Chem. 24, 1870-1882.

(30) Smith, M. E. B., Caspersen, M. B., Robinson, E., Morais, M., Maruani, A., Nunes, J. P. M., Nicholls, K., Saxton, M. J., Caddick, S., Baker, J. R, et al. (2015) A platform for efficient, thiol-stable conjugation to albumin's native single accessible cysteine. Org. Biomol. Chem. 13, 7946-7949.

(31) Sleep, D., Cameron, J., and Evans, L. R. (2013) Albumin as a versatile platform for drug half-life extension. Biochim. Biophys. Acta, Gen. Subj. 1830, 5526-5534. 
(32) Bonanata, J., Turell, L., Antmann, L., Ferrer-Sueta, G., Botasini, S., Méndez, E., Alvarez, B., and Coitiño, E. L. (2017) The thiol of human serum albumin: Acidity, microenvironment and mechanistic insights on its oxidation to sulfenic acid. Free Radical Biol. Med. 108, 952-962.

(33) Sleep, D. (2015) Albumin and its application in drug delivery. Expert Opin. Drug Delivery 12, 793-812.

(34) Yazaki, P. J., Kassa, T., Cheung, C.-W., Crow, D. M., Sherman, M. A., Bading, J. R., Anderson, A.-L. J., Colcher, D., and Raubitschek, A. (2008) Biodistribution and tumor imaging of an anti-CEA singlechain antibody-albumin fusion protein. Nucl. Med. Biol. 35, 151-158.

(35) Strohl, W. R. (2015) Fusion Proteins for Half-Life Extension of Biologics as a Strategy to Make Biobetters. BioDrugs 29, 215-239.

(36) Andersen, J. T., Cameron, J., Plumridge, A., Evans, L., Sleep, D., and Sandlie, I. (2013) Single-chain variable fragment albumin fusions bind the neonatal $F_{c}$ receptor $(F \mathrm{CR})$ in a species-dependent manner: implications for in vivo half-life evaluation of albumin fusion therapeutics. J. Biol. Chem. 288, 24277-24285.

(37) Smith, B. J., Popplewell, A., Athwal, D., Chapman, A. P., Heywood, S., West, S. M., Carrington, B., Nesbitt, A., Lawson, A. D. G., Antoniw, P., et al. (2001) Prolonged in Vivo Residence Times of Antibody Fragments Associated with Albumin. Bioconjugate Chem. 12, $750-756$.

(38) Hudis, C. A. (2007) Trastuzumab - Mechanism of Action and Use in Clinical Practice. N. Engl. J. Med. 357, 39-51.

(39) Goldstein, M. J., and Mitchell, E. P. (2005) Carcinoembryonic antigen in the staging and follow-up of patients with colorectal cancer. Cancer Invest. 23, 338-351.

(40) Holliger, P., and Hudson, P. J. (2008) Engineered antibody fragments and the rise of single domains. Nat. Biotechnol. 23, 11261136.

(41) Hudson, P. J., and Kortt, A. A. (1999) High avidity scFv multimers; diabodies and triabodies. J. Immunol. Methods 231, 177189.

(42) Morais, M., Nunes, J. P. M., Karu, K., Forte, N., Benni, I., Smith, M. E. B., Caddick, S., Chudasama, V., and Baker, J. R. (2017) Optimisation of the dibromomaleimide (DBM) platform for native antibody conjugation by accelerated post-conjugation hydrolysis. Org. Biomol. Chem. 15, 2947-2952.

(43) Matsui, S., and Aida, H. (1978) Hydrolysis of Some NAlkylmaleimides. J. Chem. Soc., Perkin Trans. 2 (0), 1277-1280.

(44) Ryan, C. P., Smith, M. E. B., Schumacher, F. F., Grohmann, D., Papaioannou, D., Waksman, G., Werner, F., Baker, J. R., and Caddick, S. (2011) Tunable reagents for multi-functional bioconjugation: reversible or permanent chemical modification of proteins and peptides by control of maleimide hydrolysis. Chem. Commun. 47, $5452-5454$

(45) Schumacher, F. F., Nobles, M., Ryan, C. P., Smith, M. E. B., Tinker, A., Caddick, S., and Baker, J. R. (2011) Situ Maleimide Bridging of Disulfides and a New Approach to Protein PEGylation. Bioconjugate Chem. 22, 132-136.

(46) Nunes, J. P. M., Morais, M., Vassileva, V., Robinson, E., Rajkumar, V. S., Smith, M. E. B., Pedley, R. B., Caddick, S., Baker, J. R., and Chudasama, V. (2015) Functional native disulfide bridging enables delivery of a potent, stable and targeted antibody-drug conjugate (ADC). Chem. Commun. 51, 10624-10627.

(47) Byeon, H. J., Min, S. Y., Kim, I., Lee, E. S., Oh, K. T., Shin, B. S., Lee, K. C., and Youn, Y. S. (2014) Human Serum Albumin-TRAIL Conjugate for the Treatment of Rheumatoid Arthritis. Bioconjugate Chem. 25, 2212-2221.

(48) Herrington-Symes, A. P., Farys, M., Khalili, H., and Brocchini, S. (2013) Antibody fragments: Prolonging circulation half-life special issue-antibody research. Adv. Biosci. Biotechnol. 4, 689-698.

(49) Todorovska, A., Roovers, R. C., Dolezal, O., Kortt, A. A., Hoogenboom, H. R., and Hudson, P. J. (2001) Design and application of diabodies, triabodies and tetrabodies for cancer targeting. J. Immunol. Methods 248, 47-66.

(50) King, D. J., Turner, A., Farnsworth, P. H., Adair, J. R., Owens, R. J., Pedley, R. B., Baldock, D., Proudfoot, K. A., Lawson, A. D. G.,
Beeley, et al. (1994) Improved Tumor Targeting with Chemically Cross-Linked Recombinant Antibody Fragments. Cancer Res. 54, 6176-6185. 